\title{
ORDERS IN SIMPLE ARTINIAN RINGS
}

\author{
BY \\ CARL FAITH $\left({ }^{1}\right)$
}

This note is a continuation of the preceding article [1]. The notation and terminology employed there will be used here.

A simple artinian ring $Q$ has the form $D_{n}$, where $D$ is a field. A subring $S$ of $Q$ is a right order in $Q$ in case $Q$ is the classical right quotient ring of $S$. Two right orders $R, S$ of $Q$ are equivalent in case there exist regular $\left({ }^{2}\right)$ elements $a, b, a^{\prime}, b^{\prime} \in Q$ such that $a R b \subseteq S$ and $a^{\prime} S b^{\prime} \subseteq R$. This relation is reflexive, symmetric, transitive, and we write $R \sim S$ (thereby suppressing $Q$ ).

The main result of [1] states that if $R$ is an order in $Q$, then for a suitable choice of a complete set $M=\left\{e_{i j} \mid i, j=1, \cdots, n\right\}$ of matrix units of $Q$, if $D$ denotes the centralizer of $M$ in $Q$, then there exists a subring $F$ of $P=R \cap D$ such that (1) $F$ is a right order in $D$, and (2) $R \supseteq F_{n}=\sum_{i, j=1}^{n} F e_{i j}$. Furthermore, we indicated by example that $R$ itself is not necessarily of the form $K_{n}$, where $K$ is an integral domain, even when $R$ possesses an identity element.

The main result of the present article states, in the notation of the paragraph above, that if $R$ is a right order of $Q$, then $R \sim P_{n}$, and, in fact, there exists a right order $U$ of $D$ such that $R \sim U_{n}$ and $U_{n} \subseteq R$ (cf. Theorem 1). Under the additional hypothesis that $R$ is a simple ring with identity, we show that $R \sim U_{n}^{2}$, and $U^{2}$ (resp. $U_{n}^{2}$ ) is a simple ring.

Henceforth, $R, Q, D, M=\left\{e_{i j} \mid, i, j=1, \cdots, n\right\}, P, F$ are fixed as in the second paragraph above, and have the same meaning as in the proof and statement of Theorem 2.3 of [1]. Two further symbols appearing there are $A=\{r \in R \mid r M \subseteq R\}$ and $B=\{r \in R \mid M r \subseteq R\}$. If $S$ is any subring, and if $X$ is a subset of $Q$, then $S[X]$ is the subring of $Q$ generated by $S$ and $X$. Throughout, the symbol $G_{n}$ denotes that $G$ is a subring of $D$, and that $G_{n}=\sum_{i, j=1}^{n} G e_{i j}$. Note that $G_{n}=G[M]$ if and only if $G$ contains the identity element of $D$.

1. TheOREM. If $R$ is a right order in the simple artinian ring $Q=D_{n}$, then:

(1) $U=B \cap P=A \cap P$ is an ideal of $P=R \cap D$.

(2) $B \cap A \supseteq U_{n} \supseteq B A \supseteq U_{n}^{2}$.

Received by the editors August 7, 1963.

(1) The author gratefully acknowledges support from the National Science Foundation under grant G-19863.

( $\left.{ }^{2}\right)$ Note that if $T$ is the classical right quotient ring of a subring, then each regular element of $T$ is invertible. Artinian rings with identity also have this property. 
(3) $P, U, U^{2}$ are right orders in $D$.

(4) $R, U_{n}^{2}, U_{n}, P_{n}$ are equivalent right orders in $Q$.

(5) If $0 \neq u \in U$, then $R\left[u^{-1}\right]=P_{n}^{\prime}$, where $P^{\prime}=P\left[u^{-1}\right]$.

Proof. (1) If $u \in U=B \cap P$, then $M u \subseteq R$ (since $u \in B$ ) and $u M=M u$ (since $u \in D$ ). Thus $u \in A$, that is, $U \subseteq A \cap P$. Similarly $A \cap P \subseteq U$, so $U=A \cap P$. Since $B$ (resp. $A$ ) is a right (resp. left) ideal of $R, U$ is a (right and left) ideal of $P$.

(2) Let $H=B \cap A$, and let $T=B A$. Since $e_{i j} B \subseteq B$ (resp. $A e_{i j} \subseteq A$ ), $i, j=1, \cdots, n$, it follows that $H \supseteq U_{n}$. Let $b \in B$, $a \in A$, let $t=b a$, and set $t_{i j}=\sum_{k=1}^{n} e_{i k} t e_{k j}, i, j=1, \cdots, n$. Since $t_{i j}$ commutes with the elements of $M$, then $t_{i j} \in D, i, j=1, \cdots, n$. Furthermore

$$
t_{i j}=\sum_{k=1}^{n}\left(e_{i k} b\right)\left(a e_{k j}\right) \in B A=T,
$$

so that

$$
t_{i j} \in D \cap T \subseteq D \cap B=P \cap B=U,
$$

$i, j=1, \cdots, n$. This shows that $t=\sum_{i, j=1}^{n} t_{i j} e_{i j} \in U_{n}$. Since each element of $T=B A$ is a sum of elements of the form $b a$, it follows that

$$
H=A \cap B \supseteq U_{n} \supseteq T=B A .
$$

Finally, we note that

$$
B A=T \supseteq H^{2} \supseteq\left(U_{n}\right)^{2} \supseteq\left(U^{2}\right)_{n},
$$

proving (2).

(3) $F$ is a right order of $D$, and $F_{n} \subseteq R$, so clearly $F \subseteq U \subseteq P$. This shows that $U$ and $P$ are right orders in $D$. Since $U^{2}$ is an ideal of $U$, it follows that $U^{2}$ is a right order in $D$, since if $d=u v^{-1}$ with $u, v \in U$, and if $0 \neq w \in U^{2}$, then $d=(u w)(v w)^{-1}$, with $u w, v w \in U^{2}$.

(4) From (3) it follows that $U_{n}, U_{n}^{2}$, and $P_{n}$ are right orders in $Q=D_{n}$. If $0 \neq u \in U$, then $u R \subseteq B$, so that

$$
u R u \subseteq B A \subseteq U_{n} \subseteq P_{n} .
$$

But $U$ is an ideal of $P$, so $u^{2} P \subseteq U^{2}$, and therefore

$$
u^{3} R u \subseteq u^{2}\left(P_{n}\right)=\left(u^{2} P\right)_{n} \subseteq U_{n}^{2} \subseteq U_{n} \subseteq P_{n} .
$$

Conversely,

$$
u^{2}\left(P_{n}\right)=\left(u^{2} P\right)_{n} \subseteq U_{n}^{2} \subseteq U_{n} \subseteq R .
$$

Since $u^{-1} \in Q$, the proof of (4) is complete.

(5) From the proof of (4) we have that $u R u \subseteq P_{n}$, so clearly $R \subseteq P_{n}^{\prime}$, and $R\left[u^{-1}\right] \subseteq P_{n}^{\prime}$. Conversely since $M u \subseteq R$, then $M \subseteq R u^{-1} \subseteq R\left[u^{-1}\right]$. 
Since $P \subseteq R$, it follows that $P^{\prime}=P\left[u^{-1}\right] \subseteq R\left[u^{-1}\right]$, and so $P_{n}^{\prime} \subseteq P^{\prime}[M]$ $\subseteq R\left[u^{-1}\right]$. This proves that $R\left[u^{-1}\right]=P_{n}^{\prime}$.

2. Theorem. If $R$ in Theorem 1 is a simple ring with identity, then:

(1) $B, A, B A=T$, and $U^{2}$ are all simple rings.

(2) $T=U_{n}^{2}$.

(3) If $0 \neq u \in U^{2}$, then $P^{\prime}=P\left[u^{-1}\right]$ and $P_{n}^{\prime}$ are simple rings.

Proof. Let $I$ be a nonzero ideal of $T=B A$. Then

$$
I \supseteq(B A) I(B A)=B(A I B) A .
$$

Since $A \cap B \supseteq U$ contains a regular element, clearly $A I B \neq 0$. Thus, simplicity of $R$ forces $R=A I B$. Therefore $I \supseteq B R A \supseteq B A$, so $B A$ is simple.

Already we have seen that

$$
T=B A \supseteq H^{2} \supseteq\left(U_{n}\right)^{2} \supseteq T^{2},
$$

where $H=A \cap B$. Since $T$ contains the integral domain $U$, then $T^{2} \neq 0$, so simplicity of $T$ yields $T=T^{2}$. It follows that

$$
T=\left(U_{n}\right)^{2}=U_{n}^{2},
$$

so simplicity of $U^{2}$ follows from that of $T$. If $0 \neq u \in U^{2}$, and if $I$ is a nonzero ideal of $P^{\prime}=P\left[u^{-1}\right]$, then $I \cap U^{2}$ is a nonzero ideal of $U^{2}$ (since $U^{2}$ is a right order in $D$ ). Thus simplicity of $U^{2}$ implies that $I \supseteq U^{2}$. Since $u \in I$ is invertible in $P^{\prime}=P\left[u^{-1}\right]$, then $I=P^{\prime}$, so $P^{\prime}$ (also $P_{n}^{\prime}$ ) is simple.

Next we show that $B$ (resp. $A$ ) is simple. Let $I$ be a nonzero ideal of $B$ (resp. A). If $0 \neq u \in U$, then $u^{3} I u \neq 0$ and $u^{3} I u \subseteq U_{n}^{2}=T$ by the proof of (4) of Theorem 1. Since $u \in B$ (resp. $u \in A$ ), it follows that $u^{3} I u \subseteq T \cap I$, so $T \cap I$ is a nonzero ideal of $T$. Simplicity of $T$ forces $I \supseteq T$. Then $I \supseteq B A B \quad$ (resp. $I \supseteq A B A)$. Since $A B=R$, then $I \supseteq B$ (resp. $I \supseteq A)$, proving that $B$ (resp. $A$ ) is simple.

3. CoRollary. Under the hypotheses of the theorem, $R=P_{n}$ if and only if $P$ is a simple ring.

Proof. The necessity is well known. Conversely if $P$ is simple, then $P=U$ by (1) of Theorem 1 . Consequently, $1 \in U \subseteq B$, so $M=M 1 \subseteq R$, and it follows that $R=P_{n}$ (since $P=R \cap D$ ).

4. Corollary. Let $R$ be a right order of $Q=D_{n}$, and assume that $R$ is a simple ring with identity element. (1) If $z$ is an element of $Q$ such that $R z \subseteq z R$, then $z$ is invertible in $Q$ and $z, z^{-1} \in R$. (2) $R$ contains the center of $Q$.

Proof. (1) Since $R$ is a right order of $Q, I=z R \cap R \neq 0$. Thus $I$ is a nonzero right ideal of $R$, and the relation $R z \subseteq z R$ implies that $I$ is an 
ideal of $R$. Since $R$ is simple, $I=R$, so $z R \supseteq R$. It follows that $z$ is not a left zero divisor in $Q$, and since $Q$ is left artinian, we conclude that $z^{-1} \in Q$. Since $R \supseteq z^{-1} R$, then $z^{-1} \in R$. Now simplicity of $R$ implies that $R z^{-1} R$, the ideal of $R$ generated by $z^{-1}$, equals $R$. Since $z^{-1} R \subseteq R z^{-1}$, we obtain that

$$
R=R z^{-1} R \subseteq R z^{-1} \subseteq R,
$$

that is, that $R z^{-1}=R$. Thus, $\left(z^{-1}\right)^{-1}=z \in R$, proving (1). (2) is an immediate consequence.

\section{REFERENCE}

1. Carl Faith and Yuzo Utumi, On noetherian prime rings, Trans. Amer. Math. Soc. 114 (1965), 53-60.

The Institute for Advanced Study, Princeton, NeW Jersey

RUtGers, The State University, NeW BrunsWick, NeW JerSeY 